\title{
Filigrane
}

Écoutes psychanalytiques

\section{L'appropriation subjective, la symbolisation, la culpabilité primaire et l'entrejeu thérapeutique : illustration clinique}

\section{Alexandre Francisco}

Volume 26, numéro 1, 2017

URI : https://id.erudit.org/iderudit/1041693ar

DOI : https://doi.org/10.7202/1041693ar

Aller au sommaire du numéro

Éditeur(s)

Revue Santé mentale au Québec

ISSN

1192-1412 (imprimé)

1911-4656 (numérique)

Découvrir la revue

Citer cet article

Francisco, A. (2017). L'appropriation subjective, la symbolisation, la culpabilité primaire et l'entrejeu thérapeutique : illustration clinique. Filigrane, 26(1),

83-95. https://doi.org/10.7202/1041693ar
Résumé de l'article

Cet article vise à illustrer les concepts psychanalytiques d'appropriation subjective, de symbolisation, de culpabilité primaire et d'entrejeu psychothérapique à travers une vignette clinique. Ces concepts s'appuient sur la pensée de plusieurs auteurs dont principalement Freud, Winnicott et Roussillon. Je ferai d'abord un bref résumé théorique des notions précédemment mentionnées. Ensuite, j’illustrerai chacun de ces concepts à travers une même vignette clinique. Concernant la patiente présentée, elle a été suivie par l'auteur dans un service hospitalier en clinique externe de psychiatrie. Certaines modifications ont été apportées afin de préserver l'anonymat de la patiente. 


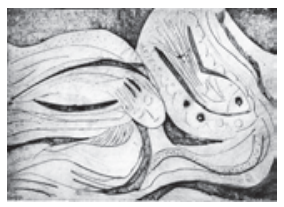

\title{
L'appropriation subjective, la symbolisation, la culpabilité primaire et I'entrejeu thérapeutique: illustration clinique
}

\author{
Alexandre Francisco
}

RÉSUMÉ: Cet article vise à illustrer les concepts psychanalytiques d'appropriation subjective, de symbolisation, de culpabilité primaire et d'entrejeu psychothérapique à travers une vignette clinique. Ces concepts s'appuient sur la pensée de plusieurs auteurs dont principalement Freud, Winnicott et Roussillon. Je ferai d'abord un bref résumé théorique des notions précédemment mentionnées. Ensuite, j'illustrerai chacun de ces concepts à travers une même vignette clinique. Concernant la patiente présentée, elle a été suivie par l'auteur dans un service hospitalier en clinique externe de psychiatrie. Certaines modifications ont été apportées afin de préserver l'anonymat de la patiente.

Mots clés: Appropriation subjective, symbolisation, vignette clinique, entrejeu, culpabilité primaire.

ABSTRACT: The goal of this article is to illustrate psychoanalytical concepts of subjective appropriation, symbolization, primary guilt and psychotherapeutic interplay. Those concepts found their support from authors such as Freud, Winnicott and Roussillon. First, I will present a briefly review those concepts and then illustrate them with a clinical case. The patient has been in psychotherapy with the author in a psychiatry outpatient clinic. Modifications have been made to preserve the anonymity of the patient.

Keywords: Subjective appropriation, symbolization, clinical case, psychotherapeutic interplay, primary guilt.

\section{L'appropriation subjective et la symbolisation}

a question de l'appropriation subjective est centrale dans le travail - de psychothérapie, mais aussi dans nos vies en général. Elle se définit comme étant la capacité de se rendre présent à une réalité interne, c'està-dire psychique, ou externe, d'en attribuer la provenance, de l'investir et de l'inscrire dans une trame historique (Jung, 2015; Fonagy et al., 2015; Roussillon, 2012; Roussillon et al., 2007). Nous sommes contraints de nous 
approprier une multitude de réalités, allant de la réalité de notre corps et de nos pulsions agressives et libidinales jusqu'à nos figures d'attachement, en passant par la réalité des limites, de l'absence, des succès, des échecs et ultimement, de la mort. Si nous ne nous approprions pas ces réalités, alors l'inapproprié fera retour dans nos vies sous différentes formes. Freud parlait de compulsion de répétition (Freud, 1920), dont la version moderne serait l'impératif à la subjectivation. Par exemple, un patient obsessionnel qui refoule son agressivité vis-à-vis de sa femme fait un lapsus en séance. Il dit: «J'ai ragé le linge de ma femme» plutôt que «ranger». Anxieux, il se défend contre cette possibilité d'être en colère contre elle; visiblement, il ne s'est pas approprié cette émotion et cette non-appropriation fait retour par un lapsus. C'est le retour du refoulé. Pensons maintenant à une patiente borderline qui en est à son quatrième conjoint violent. Elle parle des hommes comme étant tous des abuseurs, clivant ainsi ses représentations de l'autre et d'elle-même. Pourtant, en séance elle ne réalise pas qu'elle peut avoir des comportements abusifs envers son thérapeute. Elle ne s'approprie pas l'abuseur en elle et ce non-approprié fait retour par l'extérieur; il est déposé dans l'objet. C'est le retour du clivé. Prenons comme dernier exemple un patient psychotique qui dénie le décès de sa mère et qui, la nuit, rapporte qu'une entité féminine rôde dans son logement. Cette fois-ci, le non-approprié, la perte de sa mère, fait retour par l'hallucination. C'est le retour du dénié (Roussillon, 1999).

Ainsi, le meilleur moyen que l'homme a trouvé pour arriver à s'approprier toutes ces réalités est la création de symboles. De façon générale, la symbolisation est le travail psychique de transformation, de construction/ destruction, de liaison/déliaison des contenus psychiques primitifs et des expériences vécues en des contenus psychiques de plus en plus symboliques (Jung, 2015; Lecours et Bouchard, 1997; Roussillon, 2012). Cette capacité de symboliser implique aussi la conscience que l'on symbolise. C'est ce que l'on appelle la réflexivité, ou fonction réflexive, qui nous permet de considérer que nos symboles et nos représentations ne sont que perception et non pas un fait ou une vérité (Aaron, 2000; Reid, 1999; Roussillon, 2012). Un exemple de travail de symbolisation tout simple est celui du retour à la maison. Vous venez de vivre une journée au travail et vous rentrez à la maison. Votre conjoint ou conjointe vous accueille et vous racontez votre journée. Simplement un récit? Non, vous faites plus que simplement raconter votre journée. En parlant, vous transformez votre expérience vécue durant le jour en mots et en images. Vous utilisez des métaphores, exprimez des 
sentiments. Vous retravaillez vos souvenirs. Vous faites même peut-être des liens que vous n'aviez pas faits durant la journée, simplement parce que votre conjoint ou conjointe vous écoute. Vous mettez en perspective vos perceptions que vous preniez pour des faits. C'est tout un travail conscient et inconscient de liaison, de construction et de transformation. Vous reprenez votre expérience de la journée et vous vous la présentez à vous-même, vous vous en donnez une copie, grâce aux mots qui vous permettent de voir votre expérience avec une certaine distance et d'une façon différente, plus réflexive. Autrement dit, c'est un travail de transformation de l'expérience vécue en symboles. Il se peut même que ce travail de symbolisation se poursuive la nuit durant vos rêves. Bref, vous vous appropriez votre journée. Cela semble aller de soi et il est vrai que quand les choses vont bien, elles semblent se dérouler naturellement. Complexifions l'exemple maintenant.

$\mathrm{Si}$, durant la journée, vous avez vécu une épreuve très difficile, voire traumatique, que vous avez été victime d'une agression ou témoin d'un grave accident par exemple, il se peut que le récit de votre journée soit plus difficile à faire une fois à la maison. Les mots ne viendront peut-être pas si facilement, vous serez plus tendu, plus angoissé. Vous en ferez peut-être des cauchemars la nuit. Peut-être même n'arriverez-vous pas à en parler et allez-vous consommer quelques verres d'alcool. Peut-être qu'une chicane se produira entre vous et votre partenaire. Ou peut-être aurez-vous un terrible mal de tête. En d'autres mots, le travail de symbolisation, d'appropriation subjective, se fera plus difficilement qu'à l'habitude.

En fait, les symboles remplacent la réaction somatique (le mal de tête), l'agir (la fuite dans l'alcool) et l'hallucination (le cauchemar). Ils amènent aussi la création d'autres symboles (de nouveaux liens), permettent une intériorisation du conflit psychique plutôt qu'une interpersonnalisation du conflit (la chicane avec le conjoint), contribuent à une croissance psychique dans le lien intersubjectif, permettent l'apprentissage par l'expérience (Bion, 1967) et diminuent la répétition automatique de patterns intrapsychiques et relationnels (Lecours, 2007; Lecours et Bouchard, 1997). Que ce soit l'homme préhistorique qui dessinait des scènes de chasse dans sa caverne et qui tentait d'expliquer les orages par la colère d'un dieu du ciel ou qu'il s'agisse d'un enfant en train de dessiner sa famille ou encore un patient en train de se représenter différemment son problème, tous créent des symboles pour donner sens à ce qu'ils vivent afin de s'approprier toutes ces réalités. 


\section{La culpabilité primaire}

Comme je viens de le mentionner, il y a des vécus, des expériences qui peuvent être particulièrement difficiles à s'approprier, comme le sont les traumatismes. Je choisis ici une problématique que l'on retrouve souvent en psychiatrie, à savoir celle de la culpabilité primaire (Roussillon, 1999). On peut concevoir qu'il existe une culpabilité secondaire, la culpabilité œedipienne, qui est une angoisse structurante au service du développement du moi et du surmoi du sujet (Freud, 1924). Il existe par contre une culpabilité destructrice, enracinée dans un surmoi primitif, qui n'est pas au service du développement du moi, mais bien au service d'une défense massive contre des vécus traumatiques, et ce par l'utilisation d'une destruction systématique du sujet (Rosenberg, 1982). Ce type de surmoi culpabilisant à l'extrême peut faire écho à ce que Racamier (1995) appelait le «sur-anti-moi» qui fonctionne, non pas sur le mode surmoïque qui incite au renoncement pour éviter une punition, mais plutôt sur le mode de l'annihilation du moi.

Lorsque les choses se déroulent bien, le bébé cherche et préconçoit le sein et en fait la rencontre. Il vit alors une illusion cruciale: celle d'être à l'origine de sa satisfaction et donc d'être une bonne personne. C'est l'expérience du trouvé/créé (Abram, 1996; Winnicott, 1967). Cela se rapproche aussi du couple préconception/réalisation de Bion (1963). Il s'agit ainsi d'un fondement du narcissisme sain. C'est dans ces expériences que se construisent la valeur du sujet et ses représentations de lui-même (Gergely et Watson, 1996).

Mais lorsque c'est l'absence prolongée, l'abandon ou l'agression ou un peu de tous ces éléments que l'enfant rencontre, c'est alors l'illusion d'être fondamentalement mauvais qui est vécue, produisant une représentation mauvaise de soi (Fonagy, 2002). C’est comme si le bébé se disait: «Je reçois du mauvais, je dois donc être mauvais et je ne mérite que cela.» En d'autres mots, s'installera chez le sujet une culpabilité d'exister, un impératif de «rester dans le mauvais», une culpabilité primaire et destructive (Roussillon et al., 2007). Ainsi, dans cette logique paradoxale, toute forme d'amour ou de succès, le «bon» sous toutes ses formes, est impérativement exclu par le sujet, car si ce dernier se "permet» de recevoir ou de vivre de bonnes expériences, c'est la titanesque culpabilité primaire qui émerge. Du coup, nous voyons aussi la fonction défensive d'une telle culpabilité qui pourrait se formuler de la sorte: plutôt me détruire/saboter et éviter d'être coupable que d'entrer en contact avec cette culpabilité mortifère qui me renvoie aux mauvais traitements que j'ai subis. 


\section{L'entrejeu}

Les enjeux et thèmes précédemment mentionnés prennent toutes leurs couleurs et leurs forces dans ce qu'on appelle l'entrejeu de la psychothérapie (Roussillon, 2008). Il y a bien sûr la question de la compréhension clinique qui est cruciale, mais l'autre question tout aussi importante est celle de la manière dont nous intervenons auprès de nos patients. Les concepts de jeu de Winnicott (1971) et d'entrejeu de Roussillon (2008) m'apparaissent fort utiles et, à mon humble avis, incontournables si l'on veut bien aider nos patients. La capacité de jouer afin de symboliser est un accomplissement de la psyché, que ce soit le jeu des enfants ou le jeu des espaces culturels des adultes. Par contre, lors de conjonctures traumatiques, le sujet verra ses capacités de jeu très affectées; l'espace transitionnel de jeu ne se crée plus, ou que très peu (Winnicott, 1971). Ceci engendre alors des tentatives de rejouer le jeu qui a échoué. Le tout devient alors une mise en scène inconsciente de la part du patient d'une dynamique ou d'un scénario relationnel caractérisé par un conflit majeur, associé à un vécu souvent traumatique, irrésolu, et qui concerne un échec relationnel vécu avec les premières figures d'attachement. La tentative, inconsciente, du sujet de remettre en jeu ce qu'il n'a pu résoudre avec ses parents témoigne à la fois de l'espoir du sujet d'en arriver à un nouvel aménagement et à la fois des échecs qu'il a rencontrés et qu'il continue d'alimenter. La question cruciale qui se pose est alors la suivante: comment l'objet réagira et quelle sera la réponse de l'autre, en occurrence celle du thérapeute, face à cet entrejeu que lui "proposera» le patient? Le défi du thérapeute devient celui de décoder le jeu du patient et d'y participer de telle façon que cela permet au sujet de s'approprier ce qui est en souffrance de représentation. L'exemple classique est celui de la petite patiente de Winnicott (1971) qui, lors de son premier contact avec lui, plutôt que de se mettre à jouer avec des petites figurines ou de la plasticine comme le font la plupart des enfants, lui mord le doigt. Winnicott, plutôt que de se retirer, de «cadrer» sa petite patiente ou d'interpréter son geste, tolère la morsure. Il «joue le jeu» de l'agressivité. Plus tard, il lui propose une spatule qu'elle lancera vigoureusement par terre, geste qu'elle répétera souvent par la suite. Selon Winnicott, la petite avait besoin de jouer à un jeu plus primitif que le jeu normal des enfants non traumatisés, un jeu concernant la destructivité face à l'objet et la recherche d'un objet qui résiste et survit à cette destructivité (Roussillon, 2009). Cela a permis à sa petite patiente de faire une expérience, probablement insuffisamment vécue par elle, celle du jeu de l'objet qui survit à la destructivité. Du coup, la petite s'approprie quelque chose de 
très précieux: je peux haïr et l'objet survit. Ma destructivité a du bon: elle permet de me découvrir et de découvrir l'autre. C'est l'équivalent de la première chicane dans un couple après le coup de foudre: c'est éprouvant, mais quelque chose de la vraie rencontre devient possible. La question du jeu en thérapie est donc celle de la capacité du thérapeute, souvent inconsciente, de survivre psychologiquement à ces mises à l'épreuve de son patient, qui constituent des remises en scène d'expériences que le sujet a besoin de vivre, et de faire vivre, afin de s'approprier différents vécus.

\section{Illustration clinique: présentation de la patiente}

Il s'agit d'une femme d'une quarantaine d'années qui a été référée à l'urgence psychiatrique suite à une tentative de suicide. Elle a ensuite été référée en clinique externe pour une problématique dépressive, trouble de personnalité, trouble dissociatif et élément psychotique. Elle a alors débuté sa psychothérapie d'orientation psychanalytique avec moi à raison d'une séance par semaine.

Son histoire, comme celle de plusieurs patients en psychiatrie, est marquée par une enfance polytraumatisée par la négligence et les abus. Elle a été incestuée par son père pendant de longues années, sa mère ayant quitté la maison alors qu'elle n'avait pas encore 10 ans, et a vécu dans différents endroits tout au long de son enfance et adolescence. Adulte, cette instabilité s'est poursuivie où elle a par moments vécu dans la rue, fait de la prostitution et un peu de criminalité jusqu'au jour où elle a décidé d'habiter avec un homme avec qui elle passera quelques années. C'est suite au décès de celui-ci qu'elle a fait sa tentative de suicide.

\section{Illustration de l'appropriation subjective et du travail de symbolisation}

Plongeons donc dans le vif du sujet avec un thème récurrent autour duquel plusieurs enjeux se sont joués pour la patiente lors de sa thérapie. Ce thème, ce fut le sang. Au début du processus thérapeutique, elle rapportait des hallucinations de sang dans son appartement. Parfois, elle s'automutilait et rapportait étendre son sang sur ses murs. Il n'était pas toujours facile de distinguer entre la réalité et l'halluciné, mais ce qui était certain, c'est que cela donnait froid dans le dos. En fait, il semblait que j'étais davantage inquiet et effrayé qu'elle pouvait l'être. Bien qu'elle exprimât une certaine anxiété, elle paraissait plus souvent qu'autrement détachée émotivement et ce détachement allait souvent jusqu'à la dissociation. En séance, il était 
impossible dans ces moments de faire émerger quelconques associations ou liens à propos du sang. Je pouvais bien spéculer dans mon esprit mais tout ce que je pouvais faire, hormis les interventions habituelles pour s'assurer autant que possible de la sécurité de la patiente, était contenir ces états, d’y survivre en quelque sorte.

Puis, en cours de route, elle a commencé à rapporter des rêves où elle racontait être submergée de sang. Des inondations de sang, disait-elle. Toujours aucune association qui puisse permettre une élaboration quelconque. Mais je notais quand même ceci: ses hallucinations diminuèrent au fur et à mesure qu'elle rapportait ces rêves de sang. Bien que l'angoisse fût toujours aussi diffuse et l'élaboration aussi pauvre, quelque chose dans le processus de symbolisation devait bien se passer puisque j'assistais à une transformation du sang «halluciné» en du sang «rêvé».

Un peu plus tard encore, elle s'est mise à se comporter en séance comme si elle se méfiait de moi. D'un contact plutôt positif dans les premiers mois, ce dernier se changea progressivement en transfert paranoïde. Son regard devint méfiant, ses élaborations coupaient court, elle inhibait son discours. Elle exprima finalement qu'elle était convaincue de ma malveillance, qu'il ne s'agissait que d'une question de temps avant que je n'abuse d'elle, au point qu'elle songeait à ne plus venir me voir.

Pendant cette époque, elle se présenta à une séance et quelques brides associatives émergèrent enfin en lien avec le sang. À ce moment, elle s'est mise à saigner du nez en pleine séance alors qu'elle racontait une histoire d'animal torturé. De nouveaux souvenirs émergèrent concernant son père et un lieu flou où elle aurait été abusée. Tout cela restait bien précaire, mais je continuais de l'accompagner.

Par la suite, elle s'est présentée à mon bureau un matin, de toute évidence perturbée, rapportant de nouveau une terreur nocturne où le sang abondait. C'est à ce moment qu'elle fit le lien entre les couleurs sur les murs de mon bureau et les couleurs d'un endroit où elle avait été abusée, non pas par son père comme elle l'avait toujours raconté, mais par d'autres hommes à qui son père l'offrait moyennant de l'argent. Ces horribles souvenirs ont été racontés avec d'intenses affects de détresse, d'impuissance et d'effroi. Cependant, le transfert paranoïde qui s'était installé s'estompa, tout comme les hallucinations puis, plus tard, les automutilations.

Que peut-on dire de ce processus? Évidemment, plusieurs lectures sont possibles. Je vous propose de la faire sous l'angle de l'appropriation subjective et du travail de symbolisation primaire et secondaire. En effet, les 
choses se sont déroulées comme si le processus thérapeutique avait réveillé des mémoires primitives chez la patiente. On pourrait parler d'une réactivation de traces mnésiques perceptives (Roussillon, 2012) qui ont donné lieu à l'hallucination de sang, voire à du sang bien réel, sur ses murs. L'effroi que cela a suscité chez moi a donné envie de ne pas entendre ni sentir ce qu'elle me racontait. Ma capacité de survivre à ces états et à l'impuissance qui y était associée a été mise à rude l'épreuve. Mais il semble que la survivance fût suffisamment présente, car quelque chose d'important s'est produit par la suite: elle s'est mise à rêver à du sang. C'était des terreurs nocturnes et il n'y avait pas d'associations suite à ces rêves; pourtant, quelque chose semble s'être alors développé. D’une hallucination de sang, témoin de la trace mnésique perceptive, nous sommes passés à du sang sous forme d'image, sous forme de représentation de la chose, sous forme de représentation-chose. Il y aurait donc eu un travail de symbolisation primaire qui transforma la trace mnésique en représentation-chose (Roussillon, 2008).

Puis, dans une espèce de crescendo transférentiel autant sur ma personne que sur les lieux physiques de mon bureau, elle arrive à élaborer des liens avec le sang et ses nombreux vécus d'abus. Elle élabore alors ce vécu traumatique en séance. Il semble bien qu'il y ait eu passage de la représentation de la chose, le sang, vers des représentations en mots de l'expérience, à l'aide de représentations-mot. On peut alors parler d'une symbolisation secondaire qui est en fait la traduction des représentations-chose en représentations-mot (Roussillon, 2012).

De toute évidence, ce long processus a permis à la patiente de s'approprier subjectivement une partie de ses traumatismes. En se les appropriant, elle est devenue plus en contact avec son monde affectif, donnant naissance, comme elle le disait elle-même, au sentiment d'exister réellement, elle qui souffrait d'épisodes dissociatifs sévères et de symptômes psychotiques négatifs. Cela lui a aussi permis aussi de reprendre contact avec sa créativité et le monde autour d'elle. Ses traumatismes, plutôt que d'être déposés dans le monde extérieur, dans ses hallucinations et dans ses rapports paranoïdes qui la contraignaient à des agirs dangereux, se sont partiellement intégrés dans son moi, non sans souffrance.

\section{Illustration de la culpabilité primaire}

Un autre axe très important autour duquel s'est jouée la psychothérapie est celui de l'estime de la patiente. En effet, elle rapportait un narcissisme extrêmement précaire, accompagné d'auto-dévalorisation et de 
comportements de négligence, autant au plan des soins corporels, de la prise de nourriture que dans sa capacité à s'occuper de son logement. Elle pouvait dire par exemple qu'elle avait enfin trouvé une solution à ses problèmes avec un sincère enthousiasme. Questionnée sur sa solution, elle répondait, radieuse: «Je vais refaire une tentative de suicide et je ne me manquerai pas cette fois. Comme ça, je ne causerai plus de problème à personne.» À noter qu'elle ne le disait pas sur un mode de défiance ou de provocation, mais surtout sur un ton qui démontrait que s'éliminer ou se détruire allait de soi pour elle. Ses négligences sont allées jusqu'à causer des hospitalisations et des complications médicales, sans parler des nombreux auto-sabotages, ruminations suicidaires et auto-mutilations.

Malgré tout, les choses commencèrent à se stabiliser en thérapie. Dans ce chaos, des premiers signes de progrès apparurent. Mais, sans surprise, dès que les choses commençaient à bien aller pour elle, les symptômes reprenaient de plus belle. Et c'est là qu'il fut possible d'identifier progressivement un cycle qui se répéta très souvent par la suite. Nous en sommes venus ensemble à cette formule: «Quand ça va bien, ça va mal.» Et j'ajoutai pendant une séance: "On dirait presque que tout se passe comme si vous ne méritiez pas d'être bien.» Et elle de répondre: «Je ne mérite pas de vivre». Je poursuivis: "Vous ne méritez pas de vivre? C'est à croire que vous sentez tellement que vous ne méritez pas de vivre qu'il vous faudrait détruire tous vos progrès.» Nous avions alors posé un jalon important: une représentation d'elle-même tellement mauvaise, honteuse et indigne qu'elle ne méritait pas de vivre, mais aussi, par ricochet, une partie d'ellemême particulièrement destructrice qui l'attaquait de l'intérieur. Une relation d'objet avec d'une part l'agresseur qui ne tolère aucun signe d'existence et, d'autre part, l'agressée qui tente de s'effacer pour ne pas susciter les attaques de l'agresseur.

Elle mettait aussi littéralement en charpie chacune de mes «bonnes» interventions, provoquant en moi un profond sentiment de rage et de désespoir. Son ton de voix et son regard se transformaient en quelque chose de très sombre. Je me suis dit qu'elle devait me traiter, me faire sentir, comme elle avait été traitée. Je lui dis: «Lorsque vous parlez comme ça de vous, on croirait entendre votre père.» Et elle de me répondre: «C'est drôle, parfois, quand je me regarde dans le miroir, j'ai l'impression de voir mon père.» Un autre jalon se posa alors: celui de l'identification à son père. Je pus lui dire éventuellement la chose suivante: «Vous semblez vous traiter aujourd'hui comme on vous traitait enfant.» 
Cela nous amena à aller plus loin et à identifier, non pas une culpabilité dite normale, mais bien une culpabilité extrêmement intense qui se manifestait dès que la patiente commençait à se sentir exister. Aller bien signifiait une montée de la culpabilité qui suscitait une peur telle, une angoisse telle qu'elle devait s'annuler, effacer toute trace de son existence et de ses progrès pour se soulager de la culpabilité. Si elle tentait sa chance pour le bonheur, l'agresseur, le surmoi primitif et sadique, venait s'occuper de remettre le moi à sa place.

Mais aussi terrible que puisse être cette auto-destructivité, elle permet d'éviter l'appropriation des profonds sentiments de culpabilité, de honte catastrophique et des liens nocifs avec les figures d'attachement. Peu à peu, plutôt que d'agir sa culpabilité par des auto-sabotages, elle a toléré sa culpabilité. Prendre conscience de la cruauté de son père qu'elle idéalisait plutôt que de continuer à se faire du mal était en soi un progrès, mais un progrès qui a amené son lot de souffrance en termes de désidéalisation et de profondes déceptions.

\section{Illustration de l'entrejeu psychothérapique}

Reprenons un de ces moments où, comme je le mentionnais, la patiente se présentait avec un discours quasi-délirant de pessimisme et de désespoir qui était si massif et qui ne laissait tellement pas de place à autre chose que cela suscitait en moi un profond sentiment de désespoir et une forte envie de l'abandonner, de la laisser tomber et de lui faire payer tout ce qu'elle me faisait endurer. Elle disait par exemple: «Ça donne rien de venir ici. Je suis dans la merde, je vais rester comme ça et de toute façon, je suis trop vieille pour faire une thérapie et puis on s'en va nulle part avec cette thérapie là. Tu sers à rien ». Première possibilité: le thérapeute néglige son contre-transfert et sous le couvert d'un motif raisonnable «s'organise» pour terminer le processus thérapeutique. Autrement dit, le thérapeute agit. Autre possibilité, le thérapeute utilise son contre-transfert pour faire interpréter à la patiente qu'elle tente de provoquer la fin de la thérapie. Option plus intéressante que la première. Troisième option, celle du jeu. Je fais l'hypothèse que ma patiente met en jeu un scénario: celui de la patiente désespérée qui provoque le rejet et l'abandon par un autre, insensible et méchant. Je tolère puis j'entre ainsi «dans la danse» avec ma patiente. J'interviens donc mais sur un ton qui se veut suffisamment crédible pour avoir un effet mais légèrement décalé pour signifier à ma patiente que «je ne le dis pas pour vrai». Je m'avance: «Alors, si je vous comprends bien, on devrait arrêter cette thérapie là tout de suite. 
Vous avez peut-être raison: au fond qu'est-ce que ça donne? Il n'y a pas d'issue, vous êtes trop vieille, ça ne changera pas.» Surprise, la patiente se met à rire. J'en rajoute et je reprends ses mots: «Au fond, c'est pas ce que vous me dites? Je suis une merde, ça changera pas, on arrête ça ici et mettez-moi à la porte.» L'atmosphère change dans la séance. Le temps de quelques instants, j'ai personnifié ce parent abandonneur et rejetant qu'elle a en elle et dont elle ignore consciemment encore l'existence. Je deviens, par mes propos, ma mimique, ma gestuelle, un symbole de son monde interne. Elle associe d'ailleurs qu'elle se tient ce genre de discours à l'intérieur d'elle-même et que ses hallucinations auditives lui disent souvent des choses comme: «Tu ne vaux rien, abandonne tout, laisse tomber, tu n'en vaux pas la peine.» Le temps du jeu, j'ai incarné une partie d'elle, probablement un objet surmoïque primitif et sadique, qu'elle déposait en moi, que j'ai vivement senti d'ailleurs, et qui, grâce au jeu, s'est incarné et symbolisé primairement.

Ainsi donc, la métaphore du théâtre est fort utile. Quels sont le ou les personnage(s) que le patient s'approprie le moins et qu'il aura besoin de projeter, déposer, transférer sur le thérapeute? La question de l'intervention se joue ici: comment réagira le thérapeute dans ces moments? Va-t-il fuir ce personnage et tenter de maintenir le rôle du bon thérapeute? Va-t-il agir le personnage et mettre fin à la thérapie? Va-t-il interpréter? Ou bien va-t-il jouer avec le patient? Bien sûr, la question n'est pas de jouer ou d'interpréter mais bien de jouer et d'interpréter. Tout est question de dosage. Mais il m'apparaît que dans des situations telles que celles que j'ai décrites plus haut, le jeu doit précéder l'interprétation au sens où, avant de tenter d'interpréter le personnage de ma patiente, à savoir son surmoi primitif et sadique, encore faut-il qu'il puisse d'abord s'incarner dans la séance. D'où l'utilisation du thérapeute comme d'un matériau, d'un médium malléable et d'une pâte à modeler (Roussillon, 2007). Le temps du jeu, j'incarne cette partie d'elle-même devant ses yeux. D'une certaine façon, cette partie se révèle à elle grâce au jeu.

Dernier exemple: c'est une époque où elle allait bien et elle s'est présentée en séance très heureuse d'un accomplissement qu'elle avait fait, accomplissement qui s'inscrivait dans une activité ouverte au public. Spontanément, elle me tend un carton d'invitation et m'invite à cette activité pour que je puisse évidemment voir le fruit de son travail. Ma réponse fut la suivante: «Vous savez, dans le cadre du travail que l'on fait ensemble, je n'accepte pas les invitations, mais peut-être pourriez-vous me parler de ce que cela signifie pour vous de m'inviter?» Mon intervention ne facilite pas du tout 
son élaboration. Le contact semble coupé. Dans les minutes qui suivent, quelques brides associatives me font réaliser mon erreur. Je reprends: «Au fond, vous auriez bien aimé que je dise oui à votre invitation». Et c'est à ce moment que j'emploie le «ton du jeu»: «Ah, quelle bonne idée, je vais pouvoir voir ce que vous avez fait de beau, vous allez être fière, je vais être fier de vous aussi.» Elle me regarde, triste et légèrement exaspérée, et dit: «Bon, là tu as compris.» S'ensuivent des associations concernant l'abandon de sa mère et l'absence de regard positif posé sur elle pendant son enfance et surtout, cette quête inavouée de retrouver un bon parent pour elle, elle qui disait toujours être au-dessus de toutes ces considérations. Le temps du jeu, j'ai incarné ce parent tant rêvé qui serait présent dans sa vie, fière d'elle, témoin de ses accomplissements, parent évidemment cruellement absent depuis fort longtemps. Ce qui est intéressant de noter est le fait que lorsque je résiste au jeu, cela amène un appauvrissement de l'élaboration de la patiente et que lorsque je me prête au jeu, cela ouvre sur des associations et des affects chez elle. L'avantage du jeu, comme disent les enfants, est que «c'est pas pour vrai». Je ne suis évidemment pas allé "pour vrai» à l'exposition de ma patiente; par contre, nous avons mis en jeu une vraie scène psychique au service de son travail d'appropriation et de symbolisation à propos d'un autre deuil auquel elle était confrontée et qu'elle évitait de s'approprier, celui de trouver réparation à travers moi de ses blessures narcissiques d'enfance.

\section{Conclusion}

La clinique nous montre que nous nous soignons en symbolisant, dans le lien à l'autre, sans jamais de garantie de résultat. Elle nous montre aussi qu'il y a plusieurs chemins menant à la symbolisation et que nos interventions doivent être guidées par des repères qui nous aident à développer une vraie pensée clinique, au cas par cas, en tolérant l'impuissance, l'incertitude et l'incomplétude qu'implique notre métier. Cela ne semble pas très populaire à notre époque. Mais la bonne nouvelle est qu'il semble que plus nous nous approprions cette posture clinique, plus nous avons du plaisir à aider et écouter nos patients et, du coup, mieux nous les aidons. Je tiens d'ailleurs à remercier ces derniers, ainsi que mes collègues et mes stagiaires pour leur précieuse contribution.

Alexandre Francisco alex.francisco@videotron.ca 


\section{Références}

Abram, J. (1996). The language of Winnicott. London: Karnac, 2007.

Aaron, L. (2000). Self-reflexivity and the therapeutic action of psychoanalysis. Psychoanalytic psychology, 17 (4), 667-689.

Bion, W. (1963). Éléments de psychanalyse. Paris: Presses universitaires de France, 1979.

Bion, W. (1967). Réflexions faites. Paris: Presses universitaires de France, 1983.

Freud, S. (1920). Au-delà du principe de plaisir. Paris: Payot, 2010.

Freud, S. (1924). La disparition du complexe d'Edipe. Dans La vie sexuelle (p. 117-122). Paris: Presses universitaires de France, 1969.

Freud, S., (1938). De la technique psychanalytique. Paris: Presses universitaires de France.

Fonagy, P., Gergely, G., Elliot, J., et Target, M. (2002). Affect regulation, mentalization and the development of the self. New York: Other Press.

Fongay, P., Luyten, P., Allison, E. (2015). Epistemic petrification and the restoration of epistemic trust: a new conceptualization of borderline personality disorder and its psychosocial treatment. Journal of Personality Disorder, 29 (5), 575-609.

Gergely, G., et Watson, J. (1996). The social bio-feedback model of parental affect-mirroring. International Journal of Psychoanalysis, 77, 1181-1212.

Jung, J. (2015). Le sujet et son double: la construction transitionnelle de l'identité. Paris: Dunod.

Lecours, S. (2007). Supportive interventions and nonsymbolic mental functionning. International Journal of Psychoanalysis, 88, 895-915.

Lecours, S., et Bouchard, M.-A. (1997). Dimensions of mentalization: outlining levels of psychic transformation. International Journal of Pyschoanalysis, 78, 855-875.

Racamier, P.-C. (1995). L'inceste et l'incestuel. Paris: Dunod, 2010.

Reid, W. (1999). Le cadre analytique revisité. Filigrane, 8, 33-48.

Rosenberg, B. (1982). Masochisme mortifère et masochisme gardien de la vie. Dans Les cahiers de la psychanalyse et de psychothérapie, 5.

Roussillon, R. (1999). Agonie, clivage et symbolisation. Paris: Presses universitaires de France.

Roussillon, R., Chabert, C., Ciccone, A., Ferrant, A., Georgieff, et N., Roman (2007). Manuel de psychologie et de psychopathologie générale. Paris: Elsevier Masson.

Roussillon, R. (2008). Le jeu et l'entre-je (u). Paris: Presses universitaires de France.

Roussillon, R. (2009). La destructivité et les formes complexes de la survivance de l'objet. Revue française de psychanalyse, 4, 1005-1022.

Roussillon, R. (2012). Manuel de pratique clinique. Paris: Elsevier Masson.

Winnicott, D. W. (1967). Le rôle de miroir de la mère et de la famille dans le développement de l'enfant. Nouvelle revue de psychanalyse, 10, 1974, 79-86.

Winnicott, D. W. (1971). Jeu et réalité. Paris: Gallimard. 